\title{
Conhecimento e condutas dos enfermeiros na paliação oncológica
}

\author{
Knowledge and conduct of nurses in oncological palliation
}

Conocimiento y actitudes de enfermeras de paliación del cáncer

\begin{abstract}
Yasmim Simão Tenório de Melo ${ }^{1 *}$, Matheus Felipe Gonçalves de Lima Lopes ${ }^{1}$, Luanne Gomes Araújo1, Ana Clícia Delmondes Ferraz¹, Juliana Lúcia de Albuquerque Vasconcelos¹, Maria Mickaelly Salgado Sobrall ${ }^{1}$, Aléxia Silmara Pereira de Lima ${ }^{1}$, Allan Alisson da Silva ${ }^{2}$, Áurea Nunes de Oliveira ${ }^{1}$.
\end{abstract}

\section{RESUMO}

Objetivo: Descrever o conhecimento dos profissionais enfermeiros acerca de cuidados paliativos em oncologia. Métodos: Descritivo, transversal com abordagem quantitativa realizado com 17 enfermeiros assistenciais que atendem pacientes oncológicos. Realizou-se a coleta de dados por questionário estruturado elaborado pelos pesquisadores e a análise dos dados se deu por estatística descritiva. Resultados: A maior parte dos entrevistados afirmaram que a eutanásia não é vista como uma medida paliativa. Apontaram que em sua formação as disciplinas aplicadas na grade curricular em sua maioria abordam o cuidado paliativo, porém de forma superficial. Identificou a ausência da sistematização da assistência a enfermagem (SAE) e de protocolos de rotina nos serviços estudados, bem como a falta de comunicação efetiva entre a equipe de enfermagem. Conclusão: Apesar do cuidado paliativo (CP) não ser mais algo tão incomum na vivência hospitalar, ainda há escassez de debates eficazes para que as equipes de enfermagem estejam empoderadas e consigam pensar no CP como algo positivo e consiga enxergá-lo como método imprescindível na sua rotina de cuidados.

Palavras-chave: Cuidados de enfermagem, Enfermagem, Cuidados paliativos, Oncologia, Terminalidade.

\begin{abstract}
Objective: To describe the knowledge of nursing professionals about palliative care in oncology. Methods: descriptive, cross-sectional with a quantitative approach conducted with 17 nursing assistants who care for cancer patients. Data were collected using a structured questionnaire prepared by the researchers and the data was analyzed using descriptive statistics. Results: Most respondents stated that euthanasia is not seen as a palliative measure. They pointed out that in their training, the disciplines applied in the curriculum mostly dealt with palliative care, but in a superficial way. It identified the absence of systematization of nursing care and of routine protocols in the services studied, as well as the lack of effective communication between the nursing team. Conclusion: Most respondents stated that euthanasia is not seen as a palliative measure. They pointed out that in their training, the disciplines applied in the curriculum mostly dealt with palliative care, but in a superficial way. It identified the absence of systematization of nursing care (SAE) and of routine protocols in the services studied, as well as the lack of effective communication between the nursing team.
\end{abstract}

Keywords: Nursing care, Nursing, Palliative care, Oncology, Terminality.

1 Universidade Tabosa de Almeida (ASCES-UNITA), Caruaru - PE. `E-mail: yasmimsimao46@gmail.com

${ }^{2}$ Centro Universitário UniFavip, Caruaru - PE. 


\section{RESUMEN}

Objetivo: Describir el conocimiento de los profesionales de la enfermería sobre los cuidados paliativos en oncología. Métodos: Se trata de un estudio descriptivo, transversal con un enfoque cuantitativo realizado con 17 asistentes de enfermería que atender a los pacientes de cáncer. La recopilación de datos fue llevada a cabo por cuestionario estructurado preparado por los investigadores y análisis de datos era para las estadísticas descriptivas. Resultados: La mayoría de los encuestados han declarado que la eutanasia no se considera una medida paliativa. Señalaron que en su formación las disciplinas aplicadas en la tabla del plan de estudios en su mayoría se acercaron a los cuidados paliativos, pero de manera superficial. Identificó la ausencia de sistematización de los cuidados de enfermería y de protocolos de rutina en los servicios estudiados, así como la falta de comunicación efectiva entre el personal de enfermería. Conclusión: Se concluye que Apesar do CP não ser mais algo tão incomum na vivência hospitalar, ainda há escassez de debates eficaces para que as equipes de enfermagem estejam empoderadas e consigam pensar no CP como algo positivo e consiga enxergá-lo como método imprescindível na sua rotina de cuidados.

Palabras clave: Cuidados de enfermería, Enfermería, Cuidados paliativos, Oncología, Terminales.

\section{INTRODUÇÃO}

O câncer é um conjunto de mais de 100 doenças que se manifesta através de um crescimento celular em desordem, que devido a este crescimento, tem o potencial de invadir órgãos e tecidos. (INCA, MINISTÉRIO DA SAÚDE, 2018). Este conjunto de doenças são a $2^{\mathrm{a}}$ causa de morte no mundo, que implica em sofrimento desde o diagnóstico. Na maioria das vezes leva o doente de forma rápida para indicação do cuidado paliativo (CP) (ALMEIDA AR, 2019).

O cuidado paliativo é uma filosofia que tem como foco a compreensão do enfrentamento da morte como algo natural, não visa postergar a vida, mas busca alívio da dor e de outros sintomas, lança mão da necessidade constante de auxiliar o paciente nesse período tão difícil, reafirmando a valorização a vida do paciente, amparando as angústias e os medos, fornecendo suporte para que prossiga sua vida de forma mais ativa. Além disso o cuidado paliativo se estende para o olhar a família, uma vez que ela é elo do paciente, como também a equipe multiprofissional, para que possam passar pelo processo de morte e morrer fortalecidos (FLORIANO JJ, et al., 2020).

O desenvolvimento do cuidado paliativo possibilita a melhoria da qualidade de vida dos pacientes e seus familiares, por meio do diagnóstico precoce e tratamento de sintomas físicos, psicossociais e espirituais (GENEVA: WHO, 2017). Ao oportunizar um cuidado individualizado, integral, humanizado e seguro a equipe de saúde deve ser capaz de pensar criticamente sobre a sua prática, ter habilidade e conhecimento clínico específicos, devendo compreender que o cuidado é uma relação de diálogo que envolve o encontro dos seres de cuidado (SALVIANO MEM, et al., 2016).

Além da assistência em CP se embasar na humanização ela necessita ser guiada pela comunicação, que é o elemento eficaz no processo de cuidado do paciente terminal e de suma importância no tratamento. Apesar que na teoria o CP apresente todo um arsenal terapêutico baseado no respeito pelo outro, ainda é possível evidenciar na prática obstáculos para o desenvolvimento do cuidado paliativo, seja por falta de entendimento dos trabalhadores envolvidos ou carência de estrutura física ou intelectuais das instituições de saúde (FLORIANO JJ, et al., 2020).

Percebendo a importância de os profissionais estarem capacitados para prestar uma assistência eficiente, um dos profissionais que agregam grande valor na assistência é o enfermeiro. No cuidado, este membro da equipe de saúde atua como um dos profissionais que desenvolve atividades primordiais na assistência direcionada a pacientes oncológicos em $\mathrm{CP}$, dedicando grande tempo no cuidado e no contato com o paciente para estabelecimento do autoconhecimento sobre a doença e melhoria da qualidade de vida (QV) (ANDRADE FLM, et al., 2018). 
Na perspectiva de promoção da QV o enfermeiro deverá estar apto para exercer sua prática de forma autônoma, executando de forma sistematizada ações paliativas desde o diagnóstico até o CP exclusivo. Sistematizar a prática de enfermagem favorece a identificação das necessidades de cuidado manifestadas e/ou referidas pelos pacientes e familiares em sua totalidade, bem como a articulação e negociação com os demais membros da equipe de saúde em nome da concretização e melhorias do cuidado, constituindo uma estratégia adequada a uma prática centrada na pessoa e não apenas nas tarefas (SILVA MM, MOREIRA MC, 2011).

Mediante o estabelecimento da problemática relacionada a esta modalidade de cuidado e a importância do enfermeiro no contexto do desenvolvimento do cuidado paliativo este estudo teve como objetivo descrever o conhecimento de enfermeiros acerca dos cuidados paliativos em oncologia.

\section{MÉTODOS}

Trata-se de um estudo do tipo descritivo, transversal com abordagem quantitativa. A coleta dos dados foi realizada em dois serviços de saúde especializados em Oncologia localizados na cidade de Caruaru -PE, sendo um hospital (de caráter público) e um ambulatório especializado (de caráter privado e vinculado ao Sistema único de Saúde - SUS).

Participaram da pesquisa 17 enfermeiros envolvidos no cuidado aos pacientes com diagnóstico de câncer. Os profissionais foram selecionados a partir de amostragem não probabilística por conveniência, dispensando a realização de cálculo para dimensionamento da amostra. Estabeleceram-se os seguintes critérios de elegibilidade: incluídos enfermeiros vinculados aos serviços como no mínimo 2 anos de atuação e excluídos profissionais de enfermagem de formação em nível médio (técnicos e auxiliares de enfermagem).

A coleta de dados se deu através da aplicação de questionário estruturado construído pelos pesquisadores que abordava as seguintes variáveis de pesquisa: 1. A eutanásia como medida paliativa; 2. Existência de disciplinas na formação acadêmica que abordassem o cuidado paliativo; 3. Teorias de enfermagem utilizadas para melhor assistência; 4. Nível de envolvimento/integração e comunicação da equipe de enfermeiros no fornecimento dos cuidados aos pacientes; 5 . Uso de rotinas e protocolos de cuidados paliativos; 6 . Conhecimento das terapêuticas medicamentosas, suas indicações, efeitos adversos no processo de cuidar; 7. Conduta dos enfermeiros perante a abordagem familiar; 8. Conduta de enfermeiros (as) quanto a "conspiração do silêncio" como restrição e falsificação de comunicação acerca do diagnóstico e de suas perspectivas de tratamento nos cuidados paliativos.

Os dados coletados foram armazenados em um banco construído através do programa Microsoft Excel (versão 2010) e foram analisados por meio de estatística descritiva. Tais técnicas são empregadas para reunir os dados da amostra, de modo que as características do grupo de participantes serem rapidamente captadas pelo leitor (RIBEIRO AJA, 2014).

A coleta dos dados com os participantes ocorreu nos meses de novembro e dezembro de 2018 e utilizou o termo de consentimento livre e esclarecido (TCLE) de modo a considerar os preceitos éticos da pesquisa. O estudo foi aprovado pelo Comitê de Ética em Pesquisa do Centro Universitário Tabosa de Almeida ASCESUNITA tendo n.ํㅡ CAAE 99898918.8.0000.5203 como número de protocolo.

\section{RESULTADOS}

Em relação a caracterização dos 17 enfermeiros entrevistados, $88 \%(n=15)$ eram do sexo feminino e $12 \%$ $(n=2)$ do sexo masculino. Verificou-se que $71 \%(n=12)$ possuíam uma faixa etária entre 20 a 39 anos. Quanto à especialização profissional $65(n=11)$ dos entrevistados possuíam pós-graduação, sendo $18 \%(n=3)$ na área de concentração de oncologia e CP e 53\% (N=9) referente a outras especialidades.

No que se refere a avaliação das 4 primeiras variáveis que envolvem a verificação do conhecimento e condutas diretamente envolvidos na assistência de enfermagem no cuidado paliativo, encontrou-se os seguintes resultados (Tabela 1). 
Tabela 1 - Conhecimento e condutas envolvidas na paliação oncológica.

Variável

1. Eutanásia como uma medida paliativa

Variável

Sim

Não

Sim

Não

Poucas

Teoria Holística Myra Levine

Teoria do Marco conceitual - Martha Rogers

Teoria da Adaptação - Callista Roy

Nenhuma ou não registrada.

Pouco envolvimento/ integração e comunicação

Quase nenhum envolvimento/ integração e comunicação

Muito envolvimento/ integração e comunicação.

5. Utilização de rotinas e/ou protocolos de cuidados paliativos.

Sim

Não tem tempo de sentar e explicar, devido a demanda

Reserva um tempo especifico para tirar todas as dúvidas dos familiares

Não respondido ou não sabe

1588

$2 \quad 12$

$10 \quad 59$

6. Conduta dos enfermeiros perante a abordagem familiar

espeita a vontade de não expor a situação ao paciente

Nível de envolvimento/ integração e comunicação da equipe de enfermagem no fornecimento dos cuidados aos pacientes

os familiares ao acompanhamento com o profissional Psicólogo da instituição

$\begin{array}{ccc}\begin{array}{c}\text { Aconselha os familiares ao acompanhamento com o profissional Psicólogo } \\ \text { da instituição }\end{array} & 15 & 68 \\ \begin{array}{c}\text { Informa mesmo sem consentimento dos familiares a situação atual do } \\ \text { paciente }\end{array} & 0 & 0 \\ \begin{array}{c}\text { Informa mesmo sem consentimento dos familiares a situação atual do } \\ \text { paciente }\end{array} & 1 & 6 \\ \text { Não respondido ou não sabe } & 0 & 0\end{array}$

Fonte: Melo YST, et al., 2020.

paciente
Não respondido ou não sabe

$17 \quad 100$

\section{Enfermeiro na "Conspiração do Silêncio"}




\section{DISCUSSÃO}

Nas últimas décadas vem se discutindo e sendo abordado de forma frequente sobre Cuidados Paliativos, não da forma que se mude o cenário atual da maneira esperada e almejada pelos estudiosos na temática, mas quanto mais se discute sobre um tema, e este desperte inquietação, principalmente sobre cuidados no fim da vida, se alcança o objetivo que é justamente o de valorizar a vida enquanto há vida.

Quando se discute o tema eutanásia no presente artigo, e colhe-se dados onde a maioria dos enfermeiros não enxergam a eutanásia como mediada paliativa, é um resposta que se almeja de profissionais que prestam cuidados a pacientes em tratamento oncológico, por que reflete um dos princípios do Manual de Cuidados Paliativos, onde enfatiza que o CP não tem nada a ver com a eutanásia, e pensar desta forma fortalece na assistência o respeito pelo vida, e que a partir desta forma de pensar coloca o CP como método para aliviar dor, sofrimentos, medos, angústias etc. E não utilizando a eutanásia (ANCP, 2012). Empregando dados de países onde a eutanásia é legalizada, o clamor para legalizar a eutanásia não ajuda a melhorar os cuidados ao fim da vida e não resolvem a gestão dos sintomas ou aprimoram a prática dos $\mathrm{CP}$ e ainda enfatiza que essas intervenções são para $1 \%$ e não para $99 \%$ dos pacientes que estão em terminalidade (PESSINI L, SIQUEIRA JE, 2019).

Quando se obtém na pesquisa que $6 \%$ dos profissionais opinaram sobre a eutanásia como medida paliativa, apesar de ser um percentual pequeno comparado as demais respostas, é preciso dar relevância, visto que se abre uma reflexão relacionada a: Que percepção é essa voltada a pacientes oncológicos? Estes profissionais em algum momento já tiveram alguma capacitação em CP? Além de ir contra os objetivos do manual de CP, é egoísmo pensar na eutanásia como medida paliativa, e não se esforçar para que o cuidado correto, sensível, e livre de preconceitos seja colocado a frente de qualquer pensamento equivocado, relacionados a pacientes sem possibilidade de tratamento modificador da doença.

Quando se questiona sobre pensamentos, atitudes e práticas relacionadas aos pacientes sem possibilidade terapêutica, questiona-se a capacitação dos profissionais, principalmente dos profissionais que lidam diretamente com estes pacientes. No presente estudo, a maioria dos enfermeiros relataram que obtiveram disciplinas que abordaram o CP nas suas respectivas graduações, porém uma abordagem superficial sobre a temática.

Um estudo qualitativo realizado com enfermeiros que aborda o tema morte e finitude, realizado em uma UTI demonstrou que os profissionais entrevistados relataram a abordagem pouco frequente nas suas respectivas graduações, e quando colocado em pauta esta temática, era abordado de forma superficial, evidenciando a importância através das falas destes profissionais a abordagem ampla e sensível sobre os cuidados durante o processo de finitude nas instituições de ensino superior (LOPES MFGL., et al, 2020).

Percebe-se que as Instituições de ensino superior ainda são enfáticas quanto abordagem de disciplinas isoladas, que pouco se integram, desconsiderando a individualidade de cada ser humano. Ainda se prevalece a ênfase excessiva na cura, que passa a ser considerado a única finalidade de tratamento, fomentando o compromisso de cuidado quase que exclusivamente para a vida, e é para preservação desta que os estudantes se sentem capacitados (LOPES MFGL., et al, 2020).

A inclusão de temáticas sobre CP nas graduações dos estudantes de enfermagem deve ser repensada e discutida com grupos interdisciplinares compostos por professores, pedagogos, gestores institucionais e estudantes com o objetivo de analisar, avaliar, alterar e criar estratégias em face do Projeto Político Pedagógico (PPP) das Instituições de Ensino Superior (IES) (HERBERT KRN, et al., 2011). Portanto, não deve ser pensada de forma isolada, para resolução do problema visto que as disciplinas de CP não determinam como os profissionais irão atuar em suas práticas.

No cotidiano do enfermeiro, uma forma de organizar e direcionar a prática profissional é a utilização de teorias de enfermagem, que se apresenta também como uma forma de ofertar o cuidado a pacientes oncológicos de forma coerente, porque além ser a base para a prática de enfermagem, serve como um aporte 
para o referencial teórico, metodológico e prático, para que os enfermeiros (as) possam dedicar-se à construção do conhecimento (GARCIA TR, NÓBREGA MML, 2004).

Diante das respostas obtidas a maioria dos profissionais relataram que utilizam a Teoria Holística de Myra Levine. Essa teoria afirma que o ser humano é um ser holístico em contínua interação com o ambiente, em um processo de adaptação que resulta na conservação e manutenção da integridade. Teoria essa que se define por ver o homem como um "todo", dinâmico, em constante interação com o meio (ALCÂNTARA MR, et al., 2011).

A visão holística traz consigo a definição da visão de um determinado fenômeno como um todo, que leva em consideração todo os fatores que podem influenciar o fenômeno observado (LEITE TAAF, STRONG MI, 2006). Segundo Levine o estado de saúde não se restringe apenas a danos físicos, mas pode ser entendida como qualquer alteração bio-psico-social-espiritual que venha a acometer o ser humano. A enfermagem segundo o autor é vista como uma ciência humanística, de natureza holística, onde justamente o enfermeiro desenvolve o seu pensar e o cuidar em prol da manutenção da saúde das pessoas (NETO DL, NÓBREGA MML, 1999).

Observa-se outra teoria utilizada pelos enfermeiros da pesquisa, a teoria da Adaptação de Callista Roy, que também traz uma abordagem com pontos semelhantes a anterior. Segundo Roy, a pessoa é um ser social, mental, espiritual e físico, afetado por estímulos do ambiente. Portanto um ser bio-psico-social. As ações de enfermagem são realizadas de forma a englobar a pessoa como um todo, sendo assim, trata-se de um sistema adaptativo holístico, em contínua interação com o meio ambiente mutante (BARBOSA VMS, SILVA JVS, 2018).

Ambas as teorias utilizadas pelos enfermeiros atendem de forma positiva e significativa o CP em sua essência, visto que enxergar o ser humano como um ser bio-psico-social-espiritual e reconhece que todos estes aspectos podem favorecer o cuidado de forma abrangente, além de humanizar o cuidado.

Uma das formas de colocar em prática as teorias de enfermagem é utilizando a Sistematização da Assistência de Enfermagem (SAE), bem como protocolos que subsidiam a prática de enfermagem, uma vez que o planejamento e organização do cuidado como prática assistencial se formaliza através destes instrumentos. No contexto dos pacientes em CP a SAE pode favorecer a utilização do tempo disponível para a assistência de forma adequada, com objetivação, qualificação e humanização do cuidado (SILVA MM, MOREIRA MC, 2010).

Pensar que os enfermeiros da pesquisa não utilizam a SAE nem protocolos no cuidado a pacientes em $\mathrm{CP}$, uma vez que o serviço não possui, se torna uma pauta preocupante, e a partir disto pode-se questionar, como na rotina da enfermagem a utilização destes instrumentos pode ter tanta falta de credibilidade? Entretanto, a causa da não utilização destes instrumentos não pode ser apenas do hospital, uma vez que os profissionais devem ser agentes de mudanças no serviço, e cabe ao profissional colocar em evidência no seu ambiente de trabalho a SAE como um instrumento valioso para a prática de enfermagem assim, facilitando o entendimento e valorização por todos. Apesar de ser um grande desafio, a mobilização dos profissionais pode mudar o contexto atual de forma positiva.

A valorização do trabalho de enfermagem bem como dos instrumentos pelos quais a equipe de enfermagem pode utilizar no seu dia a dia, se faz através da comunicação, sendo está também uma forma valiosa de colocar em prática a confiança e o respeito entre os profissionais. Os entrevistados da pesquisa em sua grande maioria revelaram terem pouco envolvimento, interação e comunicação entre a equipe de enfermagem.

Constata-se que na assistência de enfermagem, a comunicação, interação e envolvimento são vistas como ferramentas importantes que promovem o elo entre a equipe de enfermagem, o paciente e a família, mediante o uso tanto da comunicação verbal quanto da não verbal. Mas alguns profissionais da equipe de enfermagem, apesar de reconhecerem a importância do emprego destas técnicas como recursos terapêuticos, demonstram 
não estarem preparados para atuar em situações que envolvam a comunicação na terminalidade humana (ARAÚJO MMT, SILVA, MJP, 2003).

Inserido na equipe multidisciplinar, é papel do enfermeiro atuar em prol da comunicação eficaz, aberta e adaptada ao contexto terapêutico, visando à negociação de metas assistenciais acordadas com o paciente e sua família de modo a coordenar o cuidado planejado. Nesse sentido, se a comunicação não se faz presente como ofertar um cuidado humanizado, objetivo e que proporcione respeito e sensibilidade, se não há planejamento, já que para planejar é imprescindível a comunicação entre a equipe que fortaleça esta prática (ANCP, 2012).

Quando se discute sobre respeito e sensibilidade no contexto do CP, logo pode-se levantar uma questão comum na atualidade que é a "conspiração do silêncio". O pacto do silêncio é gerado pela crença de que dizer a verdade para o paciente em cuidado paliativo é prejudicial e que o processo de decisões compartilhadas é escasso. Ainda afirma que a conspiração do silêncio traz aos familiares o sentimento de reduzir a dor do paciente frente à morte (MACHADO JC, et al., 2019).

Diante do exposto pode-se perceber sobre a importância de equipes capacitadas para o cuidar no processo de terminalidade. No presente estudo a maioria dos enfermeiros revelaram que aconselham a família a procurar acompanhamento com psicólogo (a) quando é abordado sobre não revelar o diagnóstico bem como tudo que envolve a condição do paciente para o próprio paciente. Diante disto surge possibilidades para justificar esta conduta, dentre elas: Os profissionais podem não se sentir preparados para discutir com a família a respeito ou podem não querer se envolver nestas questões ou acreditam que o profissional psicólogo (a) é o profissional apto para debater sobre esta questão. É de suma importância o envolvimento da equipe multiprofissional, e sobretudo estar preparado para se envolver enquanto parte integrante da equipe multiprofissional, inclusive o enfermeiro (a), e assim ajudar os familiares a compreender o direito e a importância da autonomia do paciente.

Poucos enfermeiros revelaram acatar a decisão dos familiares quanto a conspiração do silêncio. Mais uma vez revela-se a importância de capacitação na área de CP, uma vez que um dos objetivos dos cuidados paliativos é permitir a prevalência da autonomia da pessoa frente a uma condição de enfermidade, sua tomada de decisões é importante quando se trata da sua condição de saúde, preservando e respeitando os seus valores enquanto paciente. Portanto o conhecer sobre a finitude permite que o paciente e a família desenvolvam estratégias de luto e reforçam o sentimento de autocontrole e tomada de decisões satisfatórias (MACHADO JC, et al., 2019).

As limitações deste estudo estão voltadas para os locais onde as pesquisas foram realizadas, uma vez que não eram instituições que trabalhavam unicamente na prestação de assistência paliativa para pacientes oncológicos, uma vez que na cidade do estudo presente estudo não possui. Além de alguns profissionais se recusarem a participar da pesquisa por ser voltada ao CP, e da mudança de plantões entre os enfermeiros sem que nos fosse informado com antecedência, nos levando a encontrar os mesmos enfermeiros em dias diferentes, que inicialmente não constava na escala de profissionais das empresas nas quais o estudo foi realizado.

\section{CONCLUSÃO}

Apesar do CP não ser mais algo tão incomum na vivência hospitalar, ainda há escassez de debates eficazes para que as equipes de enfermagem estejam empoderadas e consigam pensar no CP como algo positivo e consiga enxergá-lo como método imprescindível na sua rotina de cuidados. A importância dos cursos de ensino superior se planejarem para abordar o CP é notória, para que o abordem não de forma superficial, mas de maneira em que os principais referenciais voltados a assistência de enfermagem o seja contemplado, objetivando um cuidado holístico e humanizado, empoderando o ser estudante para a prática profissional futura. Além da necessidade de implementar educação permanente nas instituições hospitalares com o intuito de disseminar o conhecimento do CP e sua aplicação na prática, de forma sensível, livre de preconceitos, julgamentos, contestações infundadas, e que deixe claro todos os direitos do paciente. 


\section{REFERÊNCIAS}

1. ALMEIDA AR. Diagnóstico de enfermagem síndrome de terminalidade: validação clínica em cuidados paliativos oncológicos. Dissertação (Mestrado Profissional em Enfermagem Assistencial) - Escola de Enfermagem Aurora de Afonso Costa. Universidade Federal Fluminense, Niterói, 2019; 127 p.

2. ANDRADE FLM, et al. Dor oncológica: Manejo clínico realizado por enfermeiros. [internet] Revista de Iniciação Científica da Universidade Vale do Rio Verde, 2018; 8(1):3-16.

3. ARAÚJO MMT, SILVA, MJP. Comunicando-se com o paciente terminal. Revista da Sociedade Brasileira de Cancerologia. [internet], São Paulo, 2003, 6(23),16-20.

4. BARBOSA VMS, SILVA JVS. Utilização de teorias de enfermagem na sistematização da prática clínica do enfermeiro: revisão integrativa. Revista de Enfermagem e atenção a saúde, Jan/Jul 2018; 7(1): 260-271.

5. BRASIL. Ministério da saúde (MS). instituto nacional do câncer (INCA), 2018.

6. FLORIANO JJ, et al. O processo de adoecer do paciente com câncer em cuidado paliativo, Revista Nursing, 2020; 23(267): 4502-4507.

7. GARCIA TR, NÓBREGA MML. Contribuição das teorias de enfermagem para a construção do conhecimento da área. Revista Brasileira de Enfermagem, 2004; 57(2):228-32.

8. HERBERT KRN, et al. "The Nurse Advocate in End-of-Life Care" The Ochsner Journal 2011:325-329.

9. LEITE TAAF, STRONG MI. A influência da visão holística no processo de humanização hospitalar. Revista o mundo da saúde São Paulo, 2006, 30 (2): 203-214.

10. ALCÂNTARA MR, et al. Teorias de enfermagem: a importância para a implementação da sistematização da assistência de enfermagem. Revista Científica da Faculdade de Educação e Meio Ambiente, 2011, 2(2):115-132.

11. LOPES MFGL, et al. Vivências de enfermeiros no cuidado às pessoas em processo de finitude. Revista Ciência Plural, $2020 ; 6(2): 82-100$.

12. MACHADO JC, et al. O fenômeno da conspiração do silêncio em pacientes em cuidados paliativos: uma revisão integrativa. Revista Enfermería Actual de Costa Rica n.36 San José Jan./Jun. 2019.

13. MANUAL DE CUIDADOS PALIATIVOS, Associação Nacional de Cuidados Paliativos (ANCP) 2ª Edição, 2012.

14. NETO DL, NÓBREGA MML. Holismo nos modelos teóricos de enfermagem. Revista Brasileira de Enfermagem, Brasília, 1999 52(2): 233-24.

15. PESSINI L, SIQUEIRA JE. Reflexões sobre Cuidados Paliativos a Pacientes Críticos em Final de Vida, Revista Bioética, Revista bioética. 2019; 27 (1): 29-37.

16. RIBEIRO AJA. Artigo Científico: Como redigir, publicar e avaliar/ Maurício [internet]. Rio de Janeiro: Guanabara Koogam, 2014. p.77, 2014.

17. SALVIANO MEM, et al. Epistemologia do cuidado de enfermagem: uma reflexão sobre suas bases. Revista Brasileira de Enfermagem, 2016; 69(6):1240-5.

18. SILVA MM, MOREIRA MC. Desafios à sistematização da assistência de enfermagem em cuidados paliativos oncológicos: uma perspectiva da complexidade. Revista eletrônica de Enfermagem, 2010, 12(3), 483-90.

19. SILVA MM, MOREIRA MC. Sistematização da assistência de enfermagem em cuidados paliativos na oncologia: visão dos enfermeiros. Revista Acta Paulista de Enfermagem [internet]. 2011;24(2):172-8.

20. WORLD HEALTH ORGANIZATION [Internet]. WHO: Definition of Palliative Care. Geneva: WHO, 2017. 